\title{
Sensor Plasmônico Kretschmann Baseado em Grafeno na Faixa de Terahertz
}

\author{
Wêndria C. da Silva, Yago G. da Conceição e Karlo Q. da Costa
}

\begin{abstract}
Resumo-Este artigo propõe um sensor de índice de refração plasmônico em terahertz baseado em grafeno. A configuração utilizada é Kretschmann, onde a variável que monitora a refletividade é o potencial químico. O sensor foi analisado teoricamente pelo método dos elementos finitos bidimensional, onde foram calculadas as refletividades e as distribuições de campo para diferentes parâmetros. Primeiramente foi feito um estudo para determinar a melhor frequência de operação, ângulo de incidência e espessura mínima da amostra. Posteriormente analisou-se a sensibilidade, largura de banda e resolução, sendo que os valores médios obtidos foram $0,312 \mathrm{eV} / \mathrm{RIU}, \mathbf{0 , 0 3 3 9 9} \mathrm{eV}$ e 0,0106 RIU, respectivamente.
\end{abstract}

Palavras-Chave-Ondas em Terahertz, Sensor Plasmônico, Grafeno, Configuração de Kretschmann.

Abstract-This paper proposes a graphene-based terahertz plasmatic refractive index sensor. The configuration used is Kretschmann, where the chemical potential is the variable that monitors the reflectivity. The sensor was analyzed theoretically by the two-dimensional finite element method, where reflectivity and field distributions were calculated for different parameters. First, a study was done to determine the best operating frequency, incidence angle and minimum sample thickness. After, the sensitivity, bandwidth and resolution were analyzed, with mean values obtained $0,312 \mathrm{eV} / \mathrm{RIU}, 0,03399 \mathrm{eV}$ and $0,0106 \mathrm{RIU}$, respectively..

Keywords-Terahertz waves, Plasmonic Sensor, Graphene, Kretschmann configuration.

\section{INTRODUÇÃo}

A plasmônica foi exposta pela primeira vez por Wood [1], em 1902 onde foi observada a emissão inconstante da luz em um espectro de difração. Antes, em 1879, Crookes [2], denominou de "matéria radiante" o quarto estado fundamental da matéria, onde íons positivos e elétrons negativos ou íons coexistem. Posteriormente, as oscilações nesse estado ionizado foram estudadas por Langmuir, o qual ele chamou de plasma [3]. Tonks e Crookes, em seguida, estudaram e verificaram que os plasmas podem desenvolver uma onda dilatacional de densidade de elétrons. Essa onda pode ser quantizada como oscilações de plasma, que pode ser chamado de plasmon [4].

As pesquisas relacionadas à plasmônica na faixa de terahertz têm crescido, e hoje têm-se diversos exemplos de aplicações. Para a frequência de THz, a área de detecção biomédica é considerada uma de suas principais aplicações. Também encontra aplicação na área de detecção biológica e de segurança, devido às suas vantagens exclusivas sobre radiações como raios $\mathrm{X}$ e raios gama, como impressões digitais não ionizantes. Os

W. C. Silva, Y. G. Conceição, K. Q. Costa, Programa de PósGraduação em Engenharia Elétrica, Instituto de Tecnologia, Universidade Federal do Pará, Belém-PA, Brasil, E-mails: wendria.cunha@gmail.com, yagogc.ee@gmail.com e karlo@ufpa.br. biossensores de Terahertz são de particular interesse, devido ao grande número de moléculas que têm seus modos vibracionais e rotacionais coletivos nessa faixa de frequência. [5]

Embora a plasmônica tenha sido estudada há bastante tempo, as tecnologias em terahertz e em plasmônica começaram a chamar atenção mais recentemente, devido suas propriedades e vantagens únicas, que possibilitam aplicação em diversas áreas [5]. Na faixa de THz, as SPW (Ondas Plasmônicas de Superfície - do inglês Surface Plasmon Wave) em metais planos não são confinadas e, portanto, não podem ser usadas para a detecção de comprimento de onda. No entanto, devido às excelentes características elétricas apresentadas pelo grafeno na região nessa faixa de frequência, viabiliza o confinamento superficial da onda plasmônica, permitindolhe, assim, a atuação em biossensores ou em dispositivos eletromagnéticos que utilizem como princípio de funcionamento a plasmônica [6].

Os sensores tradicionais baseiam-se no efeito de ressonância de plasmon de superfície (SPR), que ocorre na interface entre metais e dielétricos. No entanto, na faixa de $0,1-10 \mathrm{THz}$, os SPRs nos metais possuem baixo grau de confinamento na interface entre os dois meios, o que limita a funcionalidade desses sensores [7]. De modo a contornar esse problema, o grafeno foi utilizado como elemento base para a construção do sensor aqui proposto, por não apresentar tais limitações.

\section{Desenvolvimento TeÓRico}

O grafeno é um material formado por átomos de carbono dispostos em uma estrutura cristalina hexagonal, cuja forma é semelhante ao favo de mel (geometria bidimensional) [8], [9]. Possui excelentes características óptica, mecânica, química e eletrônica [10]. Ainda, caracteriza-se por ser bastante flexível e resistente. A sua obtenção experimental baseia-se na remoção de uma única camada de grafite, cujos métodos podem ser: esfoliação, redução de óxido de grafite, deposição de vapor químico etc., [8].

Os SPPs (Surface Plasmon Polariton) no grafeno podem ser excitados na região de terahertz, com alto confinamento espacial, baixas perdas e excelente ajuste de frequência [10]. Assim, o grafeno pode ser usado para ajustar as propriedades plasmônicas das nanoestruturas metálicas convencionais nas regiões visível e próximo ao infravermelho.

A maioria dos sensores é baseado na configuração Kretschmann no espectro de luz visível, onde o filme metálico, é depositado diretamente sobre o prisma. Na Fig. 1 é mostrado o modelo eletromagnético dessa configuração. 


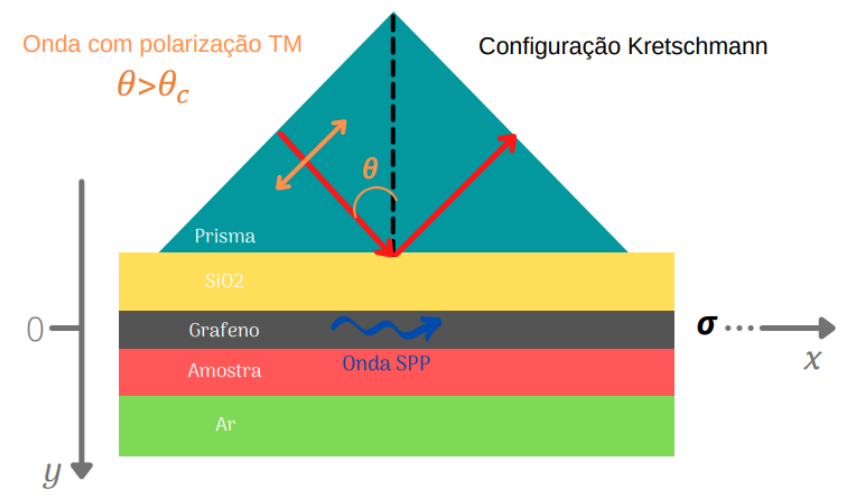

Fig. 1. A configuração Kretschmann para excitação de ondas SPP em grafeno, que está na interface do ar e $\mathrm{SiO}_{2}$, a espessura da amostra é $5 \mu \mathrm{m}$.

Na frequência de $\mathrm{THz}$, a parte imaginária da constante dielétrica do grafeno é alta, portanto, a excitação de SPPs nessa configuração necessita de um prisma de índice de refração elevado. A excitação ocorre através do tunelamento de ondas evanescentes, que originam a reflexão interna total da luz em um prisma. A permissividade do prisma é a maior das estruturas que compõem o dispositivo, a fim de produzir uma reflexão interna total da onda eletromagnética incidente [11].

Devido ao descasamento entre o momento de radiação incidente e o momento de onda SPP na folha de grafeno, não há acoplamento de ondas SPP no grafeno a partir de um feixe de onda eletromagnética incidente [11]. Essa característica existe no grafeno e nos metais. Logo, os mesmos mecanismos de excitação das ondas SPP nos metais serão utilizados no grafeno. Assim, na configuração Kretschmann, utiliza-se o método de acoplamento de prisma para excitação de ondas SPP.

O método consiste na excitação de uma onda plana incidente em um prisma cujo ângulo de incidência produz reflexão interna total, conforme mostrado na Fig. 1. Matematicamente, o ângulo de incidência $\theta$ deve ser:

$$
\theta>\theta_{c}=\sin ^{-1} \sqrt{\frac{\varepsilon_{\mathrm{SiO}_{2}}}{\varepsilon_{\text {prisma }}}}
$$

Os mecanismos de detecção do sensor em detecção espectral estão sujeitos a taxa de mudança de comprimento de onda ressonante em relação à mudança do índice de refração $(\mathrm{RI})$, onde os parâmetros são a sensibilidade (S), que é a inclinação da curva, dada por: $S\left(\Delta \mu_{c}\right)=\Delta \mu_{c} / \Delta n$ fator de qualidade (Q), figura de mérito (FOM), profundidade de modulação (MD) e full width at half maximum (FWHM). Para esse estudo foi empregado a análise do potencial químico do grafeno para substituir a modulação de comprimento de onda ou a varredura de ângulo incidente [12], [13].

A sensibilidade não define apenas a capacidade detecção e para quantificar os índices de refração das amostras, a resolução do sensor caracterizada pelo menor descolamento espectral discernível é igualmente importante, e essa resolução está fortemente ligada com MD e FWHM [12]. A resolução tem uma importância crucial no estudo desempenho de sensores pois define a capacidade do sistema de distinguir dois picos de resposta ou dois pontos com uma distância pequena entre eles [13].

\section{A. Modelagem do Sensor Proposto}

Foi utilizado um software comercial para realizar este trabalho. Através dele, é possível modelar numericamente a estrutura sob análise, por meio das seguintes etapas: construção da geometria, caracterização elétrica dos materiais presentes, definição das condições de contorno, excitação do problema e representação da estrutura através de uma malha computacional.

O modelo numérico do sensor criado no Comsol [14], foi desenvolvido utilizando uma geometria bidimensional. Isso é possível devido à simetria mostrada na estrutura do sensor, onde qualquer plano perpendicular às interfaces será sempre o mesmo. Além disso, a condição de contorno (Surface Current Density) é aplicada na folha de grafeno com o intuito de substituir essa região por uma relação de impedância apropriada entre os campos elétrico e magnético na superfície da folha. A interface física escolhida foi o modo de Rádio Frequência. Por fim, com o auxílio das equações de Maxwell, é resolvida a equação de onda, utilizando-se o método dos elementos finitos.

\section{RESUltados}

\section{A. Análise da Frequência e Ângulo}

A refletividade dos materiais é influenciada pelas variáveis do meio ambiente que está inserido, assim como o material da superfície. Portanto foram analisados frequência e ângulo, para obter os valores que terão melhor refletividade. Nas simulações (Fig. 2) o potencial químico ficou fixo em $0,5 \mathrm{eV}$ e a espessura da amostra foi definida em $h=5 \mu m$, esses valores foram usados a partir dos resultados obtidos na referência [15].

$\mathrm{Na}$ Fig. 2, nota-se que a frequência de ressonância é deslocada em direção a valores mais altos à medida que os valores de $\theta$ são aumentados, e a frequência de excitação correspondente também aumenta. Para $\theta=62^{\circ}, 72^{\circ}$ e $82^{\circ}$, as ressonâncias ocorrem em 1,66, 1, 85 e 1,97 THz, respectivamente. Nestes resultados, o potencial químico foi fixado em $\mu c=0,5 \mathrm{eV}$. Isso mostra que é possível controlar os níveis de refletividade na estrutura variando o ângulo da luz incidente $\left(\theta>\theta_{c}\right)$, para um determinado valor fixo de $\mu c$.

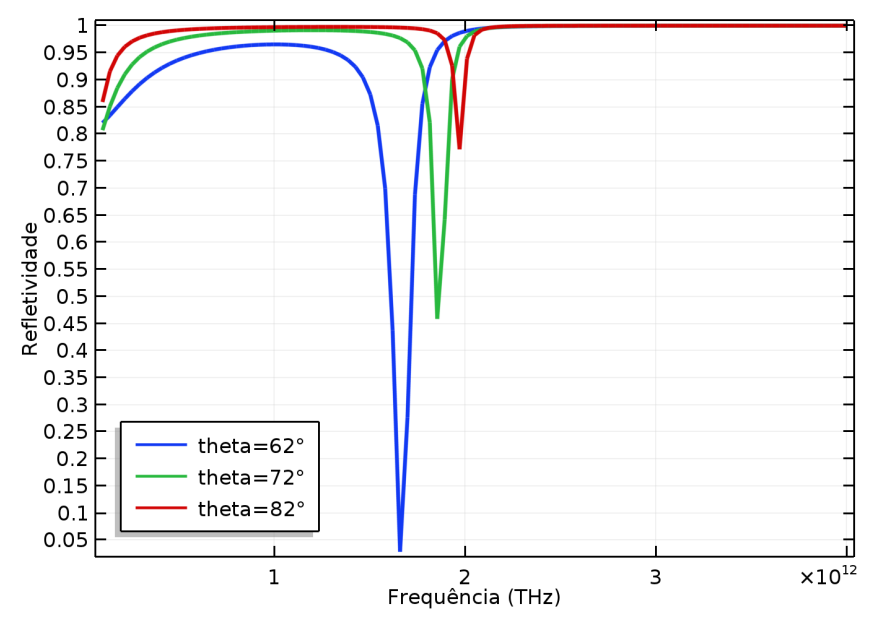

Fig. 2. Refletividade versus frequência, $\mu_{c}=0,5 \mathrm{eV}$ para a estrutura da Fig. 1, para os valores de $\theta=62^{\circ}, 72^{\circ}$ e $82^{\circ}$. 
Para cada valor de $\theta$, as ressonâncias plasmônicas no grafeno estão em frequências diferentes, onde a refletividade é mínima, a menor refletividade observada foi em 1,66 THz. Nesses pontos de mínima, as ondas SPP são excitadas. Essa queda mais acentuada no espectro de refletividade, constitui uma marca registrada da excitação do GSP (Graphene Surface Plasmons) [11].

As Figuras 3, 4 e 5 mostram a distribuição espacial da parte real da componente $z$ de campo magnético, $R e[H z]$, para cada valor de $\theta$ e sua frequência de ressonância respectiva. $\mathrm{Na}$ Fig. 3, vemos uma pequena variação na intensidade do campo magnético próximo à folha de grafeno, em ambos os lados, quando comparado com os outros casos, ilustrados nas Figuras 4 e 5 .

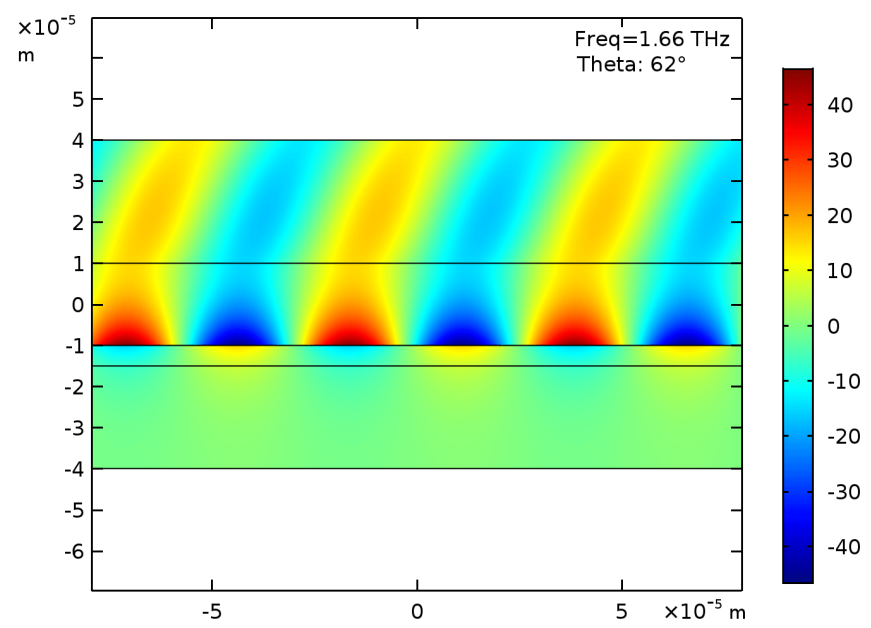

Fig. 3. Distribuição da componente de campo magnético $R e[H z]$ para 1,66 THz, $\theta=62^{\circ}$.

Essa variação está relacionada à profundidade de penetração pelicular $(\zeta)$ definida por [11], onde o resultado obtido na Fig. 3 apresenta maior $\zeta$ que o ilustrado nas Figuras 4 e 5 . Os SPPs têm a capacidade de confinar o campo eletromagnético na direção normal à interface, nas escalas de comprimento de onda abaixo do limite de difração. Essa é uma característica importante de SPPs.

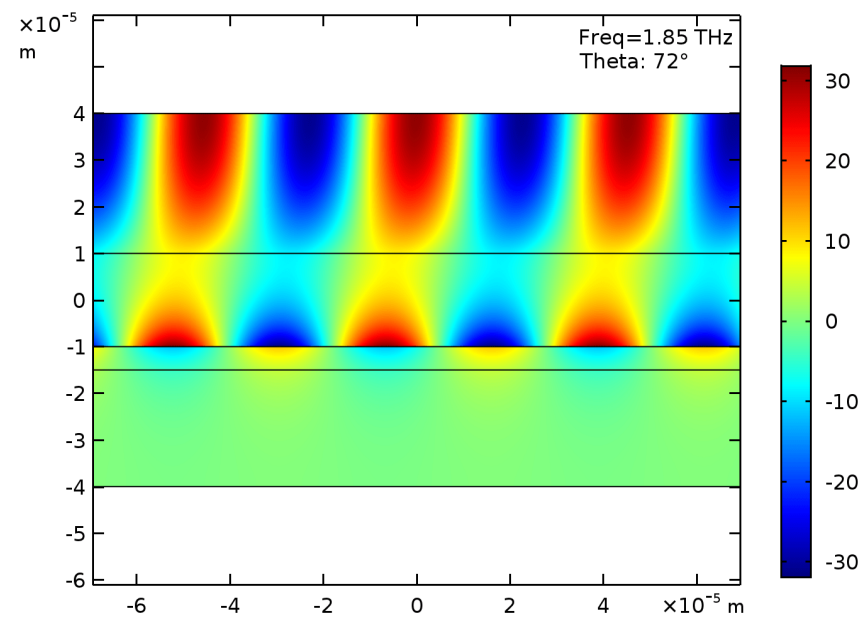

Fig. 4. Distribuição da componente de campo magnético $R e[H z]$ para 1,85 THz, $\theta=72^{\circ}$.
De acordo com [11], sabe-se que no metal, e consequentemente no grafeno, a profundidade de penetração tornase aproximadamente constante para cumprimentos de onda abaixo da frequência de ressonância plasmônica de superfície (SPR) e pode ser de várias ordens de magnitude menor que no dielétrico. Já para o dielétrico, a profundidade de penetração do campo eletromagnético aumenta de acordo com o aumento do comprimento de onda incidente [11].

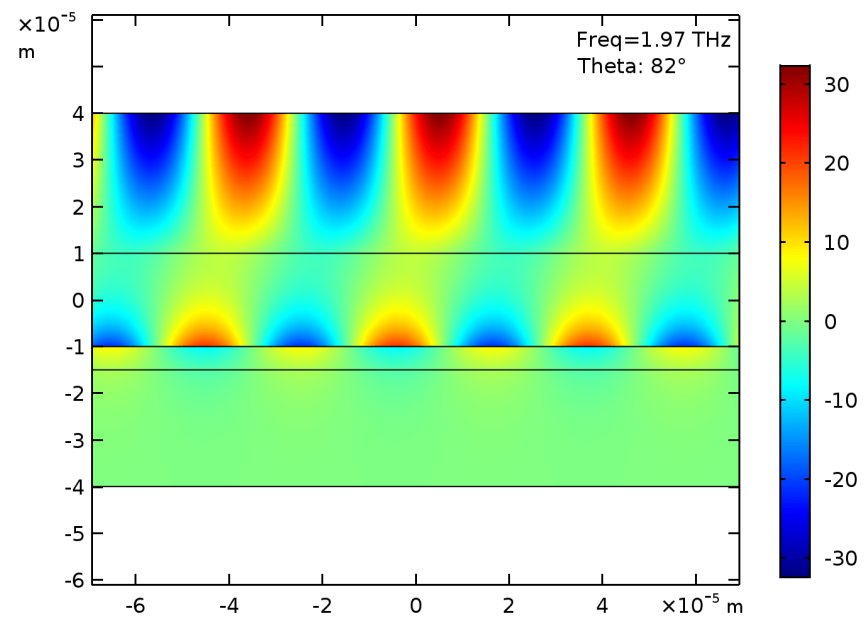

Fig. 5. Distribuição da componente de campo magnético $R e[H z]$ para 1,95 THz, $\theta=82^{\circ}$.

Portanto à medida que o comprimento de onda aumenta (frequência diminui), perde-se a capacidade dos SPPs de confinar o campo eletromagnético. Apesar do confinamento de campo ser mais fraco em frequências menores, o comprimento de propagação correspondente é maior.

\section{B. Análise da Espessura}

A SPW é muito sensível à variação do meio circundante [6]. Uma ligeira mudança no tamanho da amostra pode levar a uma mudança acentuada na curva de refletividade. Isto pode ser observado nos resultados obtidos a seguir.

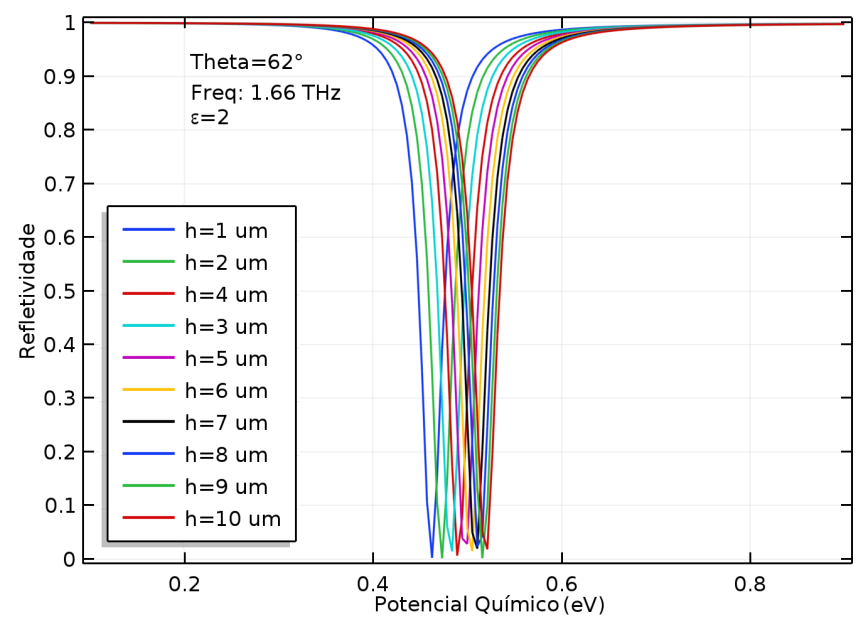

Fig. 6. Refletividade versus Potencial químico, para os valores de permissividade em $\varepsilon=2$, variando $h=1-10 \mu m$. 
Para evitar erros na detecção induzidos pela espessura da amostra, foi analisado a relação que ocorre entre as diferentes espessuras (h) e o potencial químico, para 3 permissividades genéricas $(\varepsilon=2,3$ e 4$)$, possibilitando saber a partir de qual espessura a resposta do sensor não varia mais.

Na Fig. 6 para $\varepsilon=2$, nota-se nas refletividades que a partir de $h=4 \mu m$ a tendência de comportamento das curvas é de convergir, na Fig. 7 esse comportamento começa a ser verificado a partir $h=6 \mu m$ e na Fig. 8 a partir de $h=8 \mu m$.

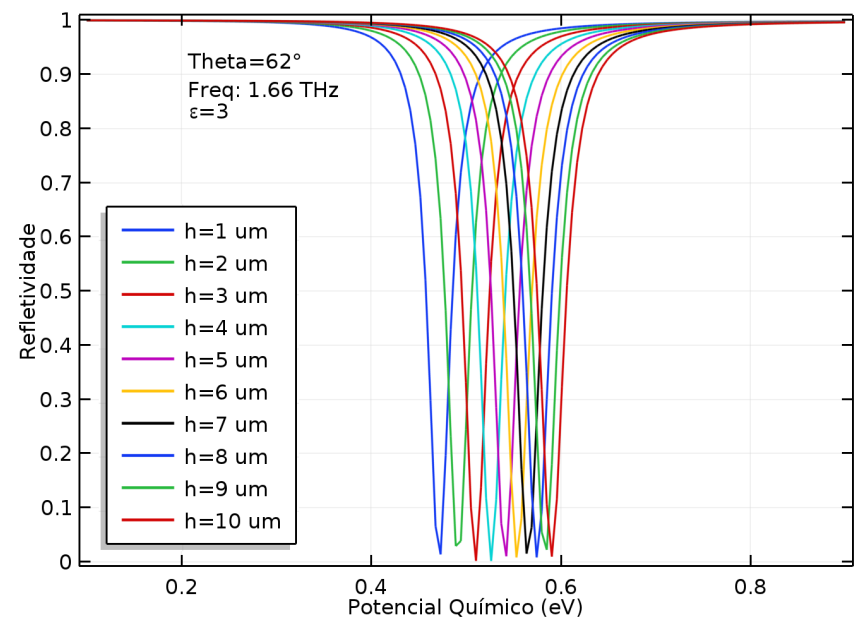

Fig. 7. Refletividade versus Potencial químico, para os valores de permissividade em $\varepsilon=3$, variando $h=1-10 \mu m$.

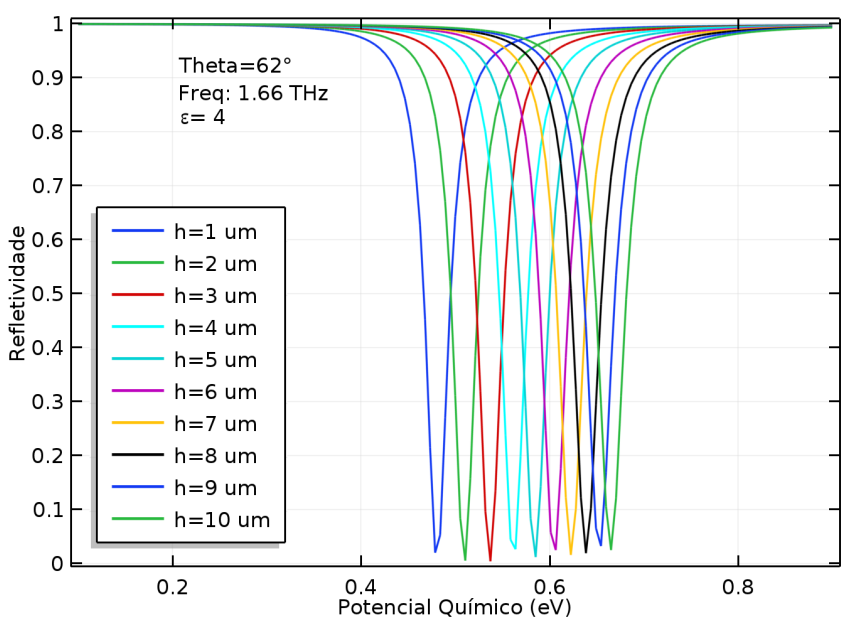

Fig. 8. Refletividade versus Potencial químico, para os valores de permissividade em $\varepsilon=4$, variando $h=1-10 \mu m$.

Na Fig. 9 podemos confirmar que a tendencia das curvas SPR é coincidir gradativamente umas com as outras à medida que a espessura da amostra aumenta para mais de $10 \mu \mathrm{m}$, portanto o local mais profundo que a onda evanescente pode penetrar é cerca de $10 \mu \mathrm{m}$. Essa rápida atenuação da onda evanescente significa que qualquer objeto fora de $10 \mu \mathrm{m}$ não afetará a medição. Logo, é possível deduzir que a altura da camada da amostra pode ser fixada para valores maiores que $10 \mu m$, pois não haverá influência na resposta do sensor.

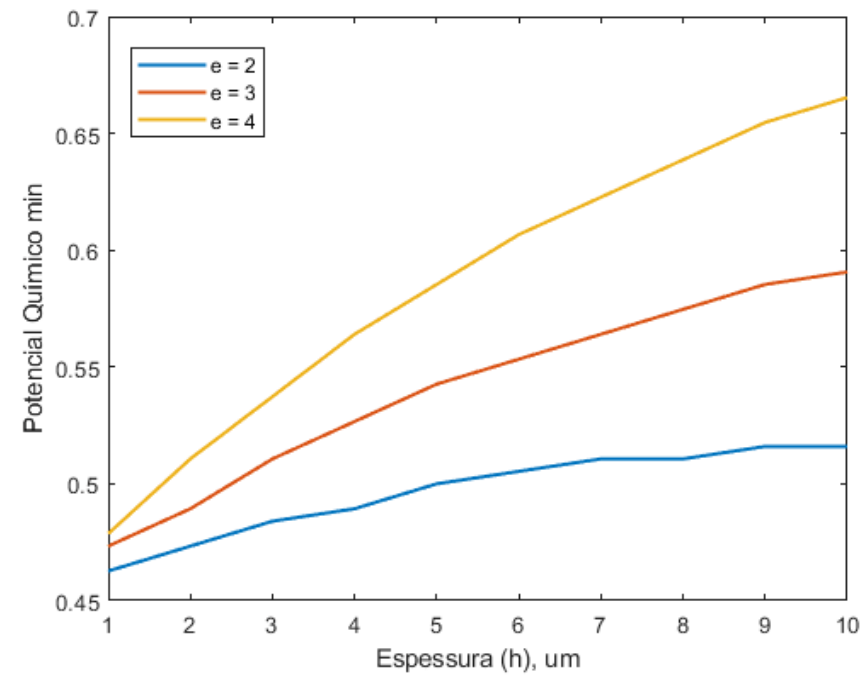

Fig. 9. Comparação das análises das refletividades das Figuras 6, 7 e 8 .

\section{Análise da Permissividade}

Ao alterarmos o potencial químico (Energia de Fermi) no grafeno, é possível detectar a menor mudança do índice de refração (raiz quadrada da permissividade), por meio da mudança na curva SPR. O extremo agudo facilita uma detecção mais fácil do deslocamento da curva de refletividade causado por uma variação sutil da permissividade do meio circundante. Portanto a análise foi feita variando a permissividade de $2-4$, com incremento de 0,1 entre elas. Podemos observar que os picos da refletividade mudam de $0,5267 \mathrm{eV}$ para $0,7052 \mathrm{eV}$ conforme a permissividade da amostra aumenta de 2 para 4 (Fig. 10).

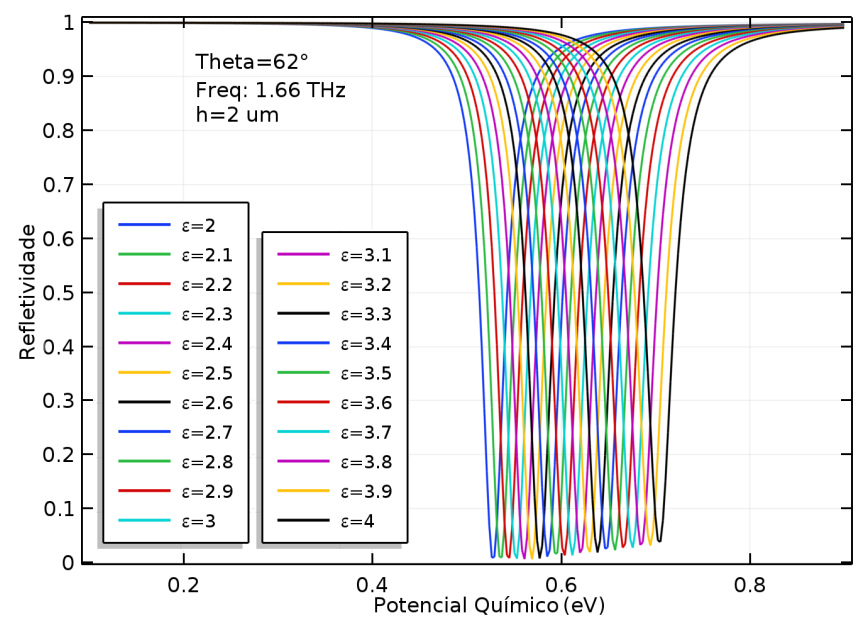

Fig. 10. Refletividade versus Potencial químico, para a frequência $1.66 \mathrm{THz}$, $\theta=62^{\circ}$ variando a $\varepsilon=2-4$.

Para poder avaliar quantitativamente a sensibilidade do sensor proposto, a Fig. 11 mostra as posições espectrais com a variação da permissividade e do potencial químico. Podemos observar também a dependência da sensibilidade com a permissividade do material. Como esperado, quanto maior a permissividade da amostra, maior será o deslocamento da curva de refletividade. 
Para avaliar o desempenho do sensor, foi calculado a sua sensibilidade média da curva em vermelho da Fig. 11 $S\left(\Delta \mu_{c}\right)=0,312 \mathrm{eV} / \mathrm{RIU}$ e para os 20 valores de permissividade em que as curvas foram geradas, foi medido o FWHM de cada uma, ele variou de 0,032 a 0,03467. Calculando a média dos resultados obtidos, temos: $0,03399 \mathrm{eV}$. A resolução do sensor é dada pela multiplicação de $S \times$ FWHM $=0,0106 R I U$. Observamos que a sensibilidade do sensor muda de forma não linear com o aumento do potencial químico à medida que o RI também aumenta, o que torna a sensibilidade dependente de RI.

O FWHM tendo um valor baixo, como neste caso, permite medir o deslocamento espectral causado pela ligeira alteração de 0.1 na permissividade amostra do sensor proposto. Aumentando, assim, a sua resolução. A resolução é definida pelo menor deslocamento espectral perceptível. A sensibilidade não determina somente a capacidade de detectar e quantificar os RI da amostra, a sua resolução é de igual importância para isto.

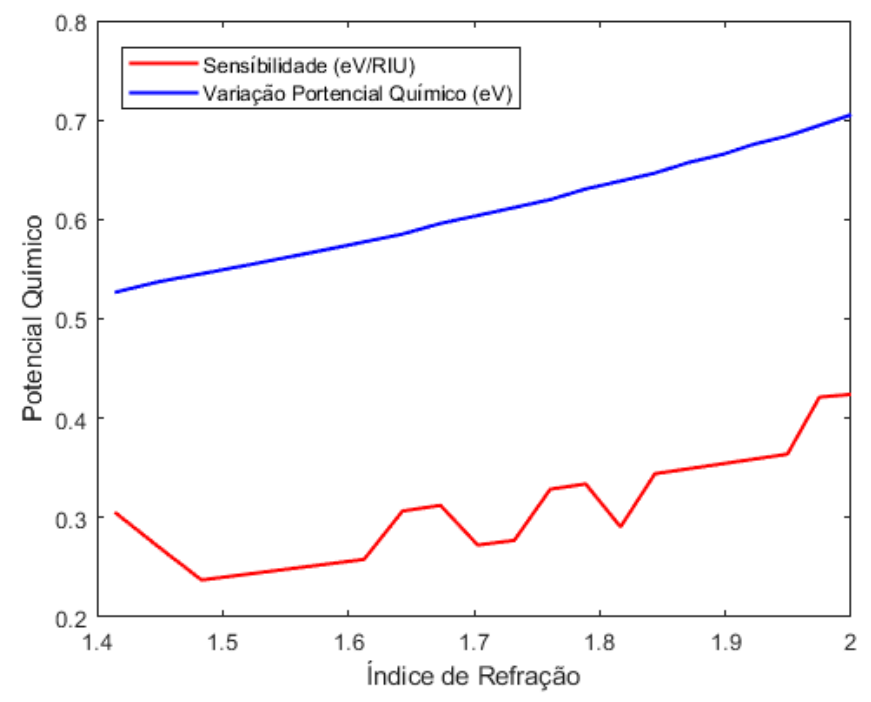

Fig. 11. Sensibilidade e variação do potencial químico em função do índice de refração do sensor proposto.

\section{CONCLUSÕES}

Neste trabalho, foi apresentado um sensor plasmônico na configuração Kretschmann baseado em grafeno. A análise numérica foi realizada pelo método dos elementos finitos (FEM), através de um software comercial para obter os resultados das curvas de refletividade para diversos parâmetros. Os resultados mostraram que o ponto de operação do sensor depende da frequência, ângulo de incidência, potencial químico da folha de grafeno, a espessura da camada da amostra e a permissividade do material.

Esses sensores usam a mudança do comprimento de onda ressonante do plasmon de grafeno como uma ferramenta para detectar pequenas mudanças no RI ambiente nas proximidades da estrutura base do grafeno. Devido à alteração do caráter metálico no grafeno, através da variação do potencial químico, a análise dessa propriedade é de fundamental importância para a inclusão desse material no projeto de sensores.
O sensor de RI mostrou boa sensibilidade e alta resolução, pois a média de FWHM é bem estreita, podendo assim, detectar desvios espectrais ocasionados por pequenas variações na permissividade entre 2 e 4 . Os valores médios obtidos da sensibilidade, largura de banda e resolução, são: $0,312 \mathrm{eV} / \mathrm{RIU}, 0,03399 \mathrm{eV}$ e $0,0106 R I U$, respectivamente. Os dados aqui estudados possibilitam a seleção de sensores específicos para otimizar sua aplicação de acordo com as necessidades de resolução e sensibilidade.

\section{AGRADECIMENTOS}

Os autores agradecem ao Conselho Nacional de Desenvolvimento Científico e Tecnológico (CNPq) e Programa de Pós-graduação em Engenharia Elétrica (PPGEE) - UFPA pelo apoio na realização deste trabalho.

\section{REFERÊNCIAS}

[1] R. W. Wood, "Xlii. on a remarkable case of uneven distribution of light in a diffraction grating spectrum," The London, Edinburgh, and Dublin Philosophical Magazine and Journal of Science, vol. 4, no. 21, pp. 396402, 1902

[2] U. Fano, "The theory of anomalous diffraction gratings and of quasistationary waves on metallic surfaces (sommerfeld's waves)," JOSA, vol. 31, no. 3, pp. 213-222, 1941.

[3] H. Zhao, Q. Guo, F. Xia, and H. Wang, "Two-dimensional materials for nanophotonics application," Nanophotonics, vol. 4, no. 2, pp. 128-142, 2015.

[4] L. Rayleigh, "Note on the remarkable case of diffraction spectra described by prof. wood (from philosophical magazine 1907)," SPIE MILESTONE SERIES MS, vol. 83, pp. 291-291, 1993.

[5] T. Globus, M. Bykhovskaia, D. Woolard, and B. Gelmont, "Submillimetre wave absorption spectra of artificial rna molecules," Journal of Physics D: Applied Physics, vol. 36, no. 11, p. 1314, 2003.

[6] Y. Xiang, J. Zhu, L. Wu, Q. You, B. Ruan, and X. Dai, "Highly sensitive terahertz gas sensor based on surface plasmon resonance with graphene," IEEE Photonics Journal, vol. 10, no. 1, pp. 1-7, 2017.

[7] J. Wu, C. Zhou, J. Yu, H. Cao, S. Li, and W. Jia, "Design of infrared surface plasmon resonance sensors based on graphene ribbon arrays," Optics \& Laser Technology, vol. 59, pp. 99-103, 2014.

[8] A. K. Geim and K. S. Novoselov, "The rise of graphene," in Nanoscience and technology: a collection of reviews from nature journals, pp. 11-19, World Scientific, 2010.

[9] H.-S. P. Wong and D. Akinwande, Carbon nanotube and graphene device physics. Cambridge University Press, 2011.

[10] Y. Zhao and Y. Zhu, "Graphene-based hybrid films for plasmonic sensing," Nanoscale, vol. 7, no. 35, pp. 14561-14576, 2015.

[11] P. A. D. Gonçalves and N. M. Peres, An introduction to graphene plasmonics. World Scientific, 2016.

[12] H. Jiang, S. Choudhury, Z. A. Kudyshev, D. Wang, L. J. Prokopeva, P. Xiao, Y. Jiang, and A. V. Kildishev, "Enhancing sensitivity to ambient refractive index with tunable few-layer graphene/hbn nanoribbons," Photonics Research, vol. 7, no. 7, pp. 815-822, 2019.

[13] A. G. Próspero, G. G. de Albuquerque Biasotti, G. A. Soares, J. V. C. Faria, R. V. R. Matos, L. A. Pinto, R. Cicolin, and J. R. de Arruda Miranda, "Aplicação de métodos matemáticos para análise de resolução espacial em sensores bac," Revista Brasileira De Física Médica, vol. 13, no. 2, pp. 43-46, 2019.

[14] C. INC., "Comsol multiphysics® software for multiphysics simulation," 2021.

[15] W. C. da Silva, R. R. Paiva, G. T. de Sousa, and K. Q da Costa, "Graphene-based terahertz plasmonic sensor," in 2019 SBMO/IEEE MTT-S International Microwave and Optoelectronics Conference (IMOC), pp. 1-3, IEEE, 2019. 